\title{
Survival of lethal poxvirus infection in mice depends on TLR9, and therapeutic vaccination provides protection
}

\author{
Christofer Samuelsson, ${ }^{1}$ Jürgen Hausmann, ${ }^{1}$ Henning Lauterbach, ${ }^{1}$ Michaela Schmidt, ${ }^{1}$ \\ Shizuo Akira, ${ }^{2}$ Hermann Wagner, ${ }^{3}$ Paul Chaplin, ${ }^{1}$ Mark Suter, ${ }^{1,4}$ \\ Meredith O'Keeffe, ${ }^{1}$ and Hubertus Hochrein ${ }^{1}$
}

\begin{abstract}
${ }^{1}$ Department of Research, Bavarian Nordic, Martinsried, Germany. ${ }^{2}$ Department of Host Defense, Research Institute for Microbial Diseases, Osaka University and ERATO, Osaka, Japan. ${ }^{3}$ The Institute of Medical Microbiology, Immunology and Hygiene, Technical University of Munich, Munich, Germany. ${ }^{4}$ University of Zurich, Zurich, Switzerland.
\end{abstract}

\begin{abstract}
Poxviruses such as the causative agent of smallpox have developed multiple strategies to suppress immune responses, including the suppression of DC activation. Since poxviruses are large DNA viruses, we hypothesized that their detection by DCs may involve the endosomal DNA recognition receptor TLR9. Indeed, we have shown here that DC recognition of ectromelia virus (ECTV), the causative agent of mousepox, completely depended on TLR9. The importance of TLR9 was highlighted by the fact that mice lacking TLR9 showed drastically increased susceptibility to infection with ECTV. In contrast, we found that the strongly attenuated poxvirus modified vaccinia virus Ankara (MVA) activated DCs by both TLR9-dependent and -independent pathways. We therefore tested whether we could use the broader induction of immune responses by MVA to protect mice from a lethal infection with ECTV. Indeed, MVA given at the same time as a lethal dose of ECTV protected mice from death. Importantly, MVA also rescued TLR9-deficient mice if administered 2 full days after an otherwise lethal infection with ECTV. Therefore, these data suggest an essential role for TLR9 in the defense against poxviruses. In addition, postexposure application of MVA may protect against lethal poxvirus infection.
\end{abstract}

\section{Introduction}

Poxviruses, including variola virus (VARV), the causative agent of smallpox, are highly pathogenic dsDNA viruses. It is proposed that smallpox has caused more death within the human population than any other infectious disease so far (1). Even though traditional vaccination programs have eradicated VARV as a natural pathogen, enhancing the knowledge of mechanisms of poxvirus infections and/or protection remains essential given the scenarios of zoonotic poxvirus infections (e.g., monkeypox), the reemergence of VARV by accidental release, or the possibility of terrorist attacks with poxviruses.

The genus Orthopoxvirus contains several related viruses based on genetic similarity and immunological cross-reactivity, including VARV, ectromelia virus (ECTV), cowpox virus (CPXV), monkeypox virus (MPXV), camelpox virus (CMPV), and vaccinia viruses (VACVs). The VACVs include the highly attenuated modified VACV Ankara (MVA), which has lost its ability to replicate in mammals. Among orthopoxvirus infections, ECTV infection of mice closely models VARV infection in humans, since it is a species-specific virus infecting its natural host and can cause fatal outcomes after respiratory inoculation with low virus doses $(1,2)$.

Nonstandard abbreviations used: $\mathrm{CDC}$, conventional DC; $\mathrm{CNPV}$, canarypox virus; CPXV, cowpox virus; CVA, VACV strain Ankara; ECTV, ectromelia virus; FL, flt3 ligand; GM, GM-CSF; HSV, herpes simplex virus; IFN-I-R, IFN-I receptor; MPXV monkeypox virus; MVA, modified VACV Ankara; pDC, plasmacytoid DC; SFV, Shope fibroma virus; $\mathrm{TCID}_{50}, 50 \%$ tissue culture infective dose(s); $\mathrm{VACV}$, vaccinia virus; VARV, variola virus.

Conflict of interest: C. Samuelsson, J. Hausmann, H. Lauterbach, M. Schmidt, P. Chaplin, M. Suter, M. O’Keeffe, and H. Hochrein were employees of Bavarian Nordic GmbH.

Citation for this article: J. Clin. Invest. 118:1776-1784 (2008). doi:10.1172/JCI33940.
Poxviruses have evolved multiple strategies for immune suppression, among which are soluble cytokine and chemokine receptors and a multitude of molecules that interfere with intracellular signaling cascades (3). Recently, molecules expressed by poxviruses have been shown to target members of the TLR signaling cascade, suggesting a role for TLR-dependent recognition pathways in the recognition of poxviruses (4). In fact, a role for TLR2 in the recognition of VACV was proposed (5). Other members of the TLR family include endosomally located receptors that recognize nucleic acids. One of these, TLR9, recognizes DNA, including synthetic oligonucleotides, bacterial and viral DNA, and under some circumstances even mammalian DNA $(6,7)$. We and others have previously shown that dsDNA viruses such as herpesviruses or adenoviruses can also be detected via TLR9, but potent alternative viral recognition pathways exist, possibly explaining why viral infection studies shown so far demonstrate no or only mild increases in susceptibility in the absence of TLR9 $(5,8-13)$. We hypothesized that since poxviruses are large DNA viruses, the TLR9-dependent recognition of the viral DNA might also play a role in poxvirus recognition.

The activation of DCs and the release of antiviral cytokines including IFN- $\alpha$ is a key component of the innate immune response. Since DCs are also the professional antigen presenters of the immune system, their activation is extremely important in the induction of adaptive immune responses.

In this study we tested the activation and the requirement for TLR9 of both plasmacytoid DCs (pDCs) and conventional DCs (cDCs) upon infection with the attenuated MVA or pathogenic ECTV orthopoxviruses. MVA activated both pDCs and cDCs and induced their production of cytokines (e.g., IFN-I) in vitro via both TLR9-dependent and -independent pathways. However, ECTV only activated $\mathrm{pDCs}$ and only via TLR9. Thus, we confirm previous 

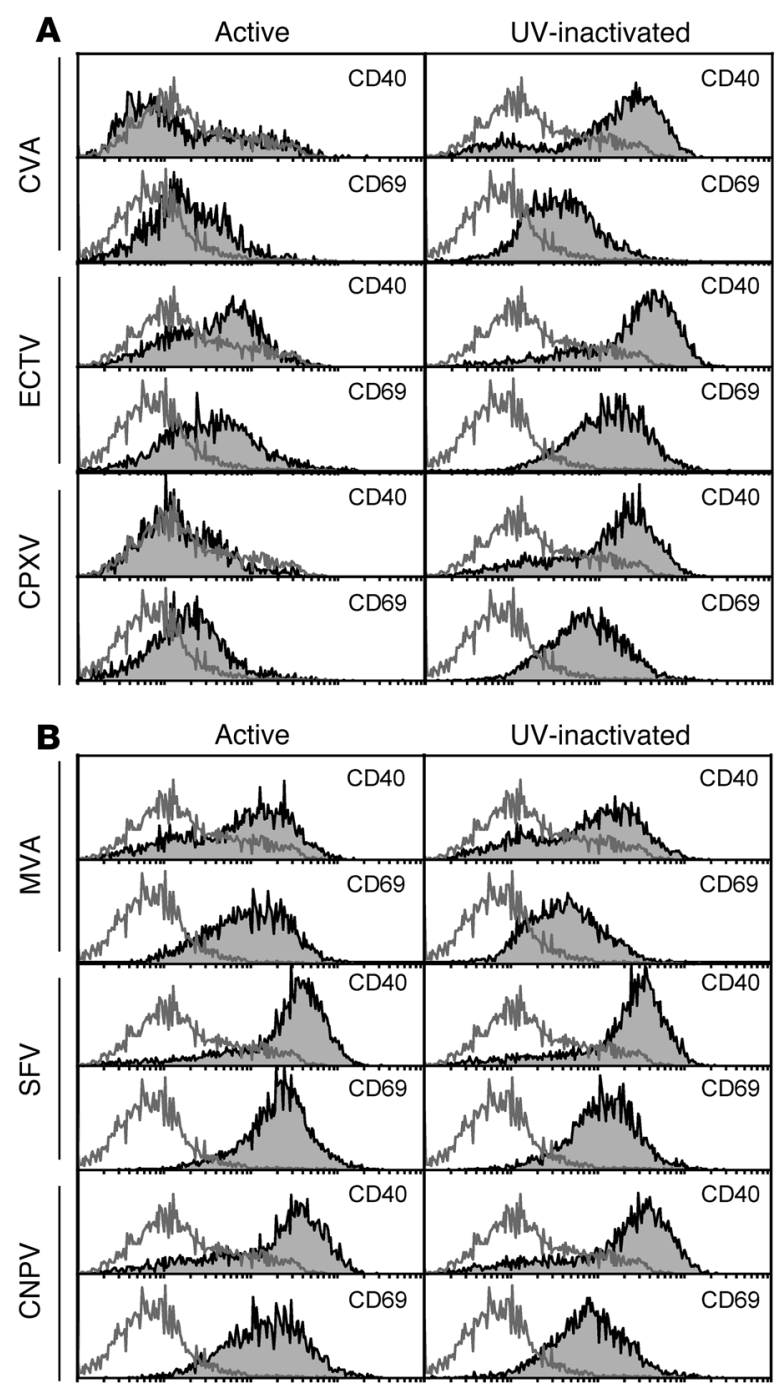

data that showed members of the poxvirus family are recognized via TLR-independent recognition pathways $(5,14)$. However, for the first time to our knowledge, we show that poxviruses are also seen via the TLR9-dependent pathway.

The in vivo relevance of this TLR9-only dependence for ECTV recognition was clearly illustrated by our in vivo studies that revealed that the lack of TLR9 rendered mice more than 100-fold more susceptible to infection with ECTV. A high susceptibility to ECTV was also shown in mice unable to respond to IFN-I due to the lack of an essential receptor chain and Rag1 $1^{-/}$mice that lack all $\mathrm{B}$ and $\mathrm{T}$ cells. This confirms previous data indicating that the survival of pathogenic poxvirus infections depends on innate as well as adaptive immune responses.

Due to the fact that traditional vaccination with MVA protects against other orthopoxvirus infections and induced in vitro TLR9dependent and -independent immune activation, we hypothesized that MVA may be used to protect mice against infection with ECTV. Administration of MVA at the same time as high doses of ECTV protected WT mice and those lacking TLR9 from death. Mice without a functional IFN-I response were protected to low and intermediate ECTV challenges; however, they succumbed to infection if higher doses were used. Mice lacking adaptive immune responses

\section{Figure 1}

Analysis of DC maturation in response to active or inactivated poxviruses. Flow cytometry histograms showing expression of CD40 or CD69 on FL-DCs after incubation with active (left) or UV-inactivated (right) poxviruses (filled histograms) in CVA, ECTV, or CPXV (A) or MVA, SFV, or CNPV (B) or without stimulation (open histograms). Results of 1 representative experiment of at least 3 (CVA, ECTV, MVA) or 2 (CPXV, SFV, CNPV) are shown.

(Rag1 $1^{-/}$mice) had some temporary survival advantage with MVA administration, but all mice eventually died, indicating that the induction of adaptive immune responses are essential for the overall protection to lethal poxvirus infection. Importantly, even postexposure application of MVA protected TLR9-deficient mice from death against prior inoculation with a lethal dose of ECTV.

This study demonstrates for the first time to our knowledge that TLR9 is an important and in vivo highly relevant pattern recognition receptor for the recognition of and the defense against pathogenic poxviruses. Moreover we demonstrate that even under certain conditions of compromised immunity, MVA activates and bridges innate and adaptive immune responses, resulting in both long-lasting and, importantly, immediate and therapeutic protection against lethal poxvirus challenge.

\section{Results}

Inactivation of VACV, CPXV, and ECTV but not of MVA, canarypox virus, and Shope fibroma virus increases DC maturation. Previously it has been described that several strains of VACV inhibit the maturation of cDCs, whereas maturation occurred in response to $\operatorname{MVA}(15,16)$. Since these studies analyzed only the role of cDCs in the absence of pDCs, we employed Flt3 ligand-generated murine DCs (FL-DCs), which consist of DCs that closely resemble ex vivo mouse spleen $\mathrm{cDCs}$ and $\mathrm{pDCs}(17)$. To test whether different stimulatory activities of different VACVs were due to lack of stimulus or active inhibition by a virus-encoded component, we incubated FL-DCs with several different strains of poxviruses either as active virus or after UV inactivation. The activation of DCs in response to VACV strain Ankara (CVA), ECTV, and CPXV was amplified after viral UV inactivation compared with the activation of DCs in response to active viruses (Figure 1A). These initial data indicated that an inhibitory component, acting on DCs, was made by those viruses. In contrast, DC activation as measured by the upregulation of CD40, CD69, and CD86 in response to MVA as well as the canarypox virus (CNPV) and rabbit Shope fibroma virus (SFV) was not increased after UV inactivation (Figure 1B and data not shown), suggesting that these viruses lacked an active inhibitory component. The production of cytokines including IFN- $\alpha$ and IL- 6 increased after the UV inactivation of CVA, ECTV, and CPXV but not of MVA, CNPV, and SFV, suggesting a broad inhibition of viral recognition and DC function, not restricted to maturation (data not shown).

Recognition of ECTV but not of MVA depends exclusively on TLR9. We have previously shown that dsDNA viruses such as herpesviruses or adenoviruses could be recognized via TLR9-dependent as well as TLR9-independent recognition pathways $(8,12)$. To elucidate the role of TLR9 in the recognition of poxviruses, we generated FL-DCs of WT or TLR9-deficient animals. CVA did not induce solid maturation of DCs from either WT or TLR9-deficient mice. However, ECTV induced some DC maturation in WT DCs, but none in the absence of TLR9, as monitored by the lack of upregulation of CD40 and CD69, indicating a strong depen- 

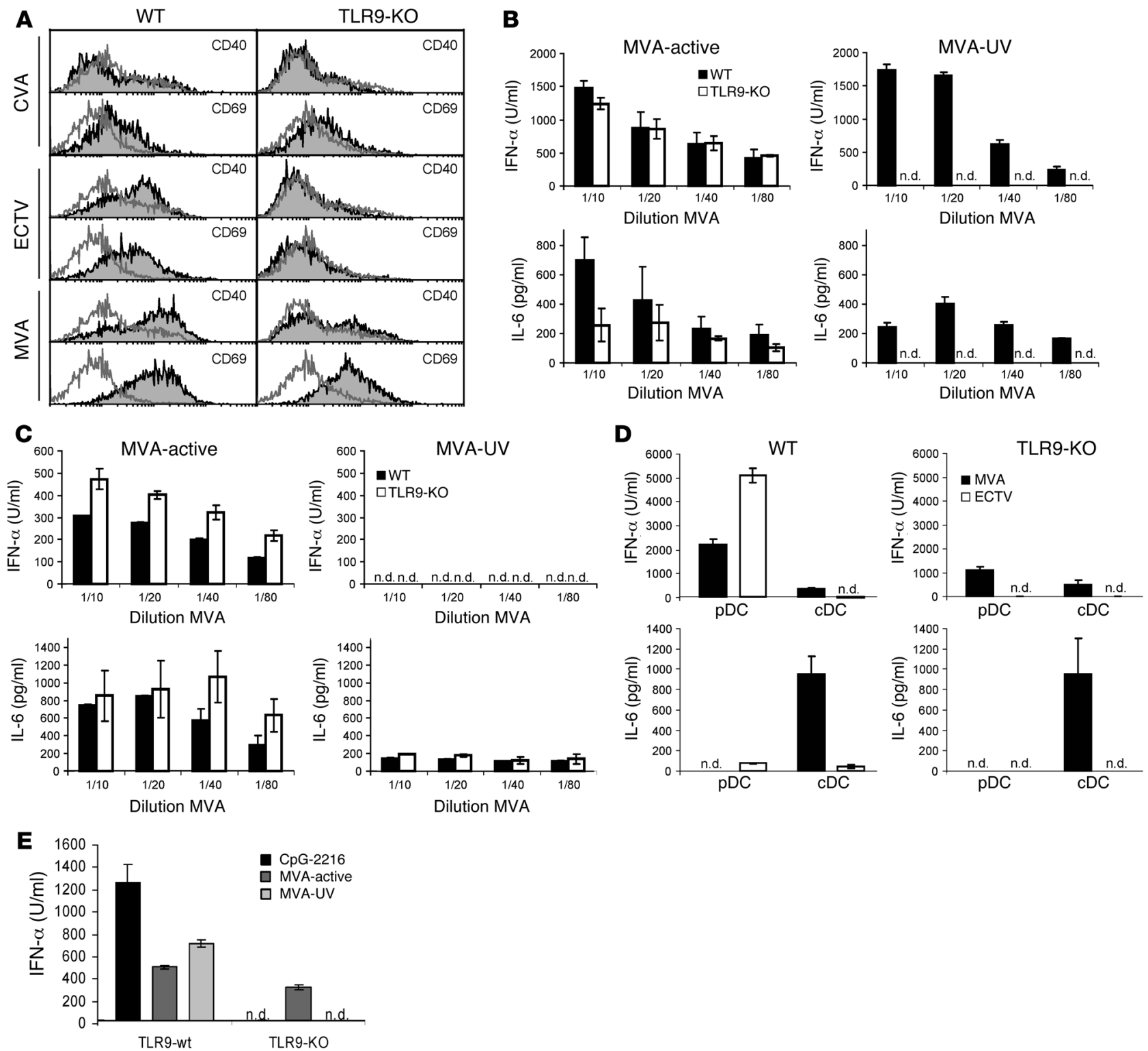

Figure 2

Response of TLR9-deficient or WT cells to poxvirus infection in vitro. (A) Flow cytometry histograms showing expression of CD40 or CD69 on FL-DCs of WT (left) or TLR9-deficient mice (right) after incubation with CVA, ECTV, or MVA as indicated (filled histograms) or without stimulation (open histograms). The histograms shown for WT are the same as those in Figure 1. FL-DCs (B) or GM-DCs (C) of TLR9-deficient (white bars) or WT mice (black bars) were stimulated with active (left) or UV-inactivated (right) MVA, and the supernatants were analyzed for IFN- $\alpha$ and IL-6 by ELISA. (D) Sorted FL-pDCs and cDCs of TLR9-deficient or WT mice as indicated were stimulated with MVA (black bars) or ECTV (white bars), and supernatants were analyzed for IFN- $\alpha$ and IL-6 by ELISA. (E) Total bone marrow cells were stimulated with active (dark gray bars) or UV-inactivated (light gray bars) MVA or CpG-2216 (black bars), and the supernatants were analyzed for IFN- $\alpha$ by ELISA. Representative results of at least 2 experiments are shown. Data represent mean \pm SD. n.d., not detected.

dence upon TLR9 for the recognition of ECTV. In contrast, in the absence of TLR9, MVA induced robust upregulation of CD69 but a drastically reduced upregulation of CD40 (Figure 2A), suggesting that the response to MVA is based on both TLR9-independent and TLR9-dependent recognition events.

FL-DCs contain both pDCs, known as the sole cell type producing large amounts of IFN- $\alpha$ in response to TLR9 activation, and cDCs, known to be unable to produce large-scale IFN- $\alpha$ production in response to TLR9. FL-DCs of WT and TLR9-deficient mice were incubated with active MVA and produced dose-dependent robust amounts of IFN- $\alpha$ and IL- 6 , demonstrating the existence of a TLR9-independent recognition pathway for MVA (Figure 2B). However, UV-inactivated MVA induced IFN- $\alpha$ and IL- 6 solely in WT and not in TLR9-deficient FL-DCs, reinforcing the notion suggested by the maturation data, that the recognition of MVA also employed a TLR9-dependent component (Figure 2B).

DCs generated in vitro with GM-CSF result in a DC population (GM-DCs) that is able to produce IFN- $\alpha$ in response to active DNA 

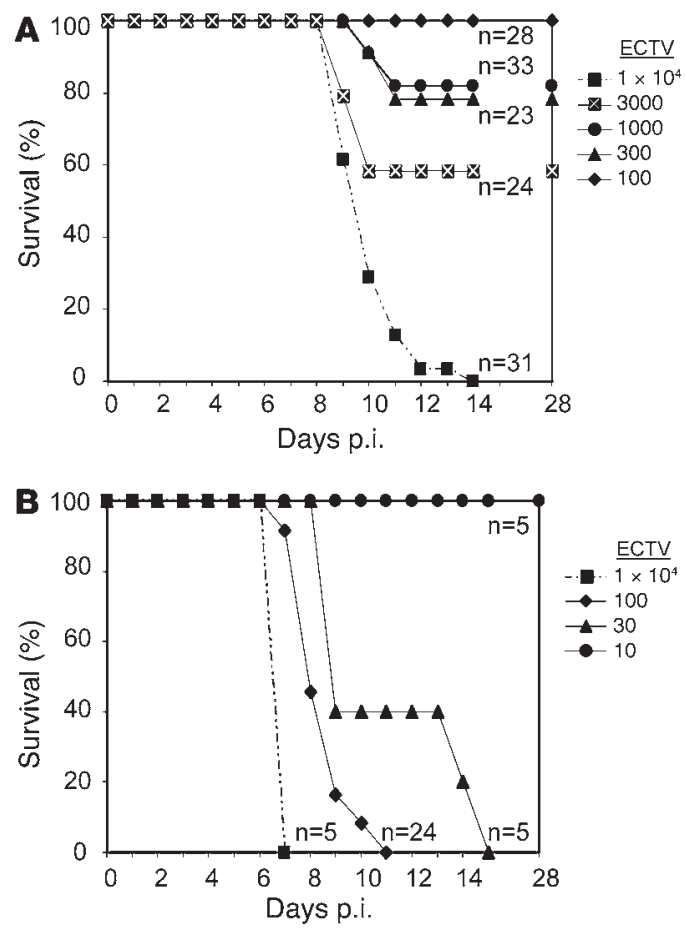

viruses (e.g., herpes simplex virus [HSV]) but not to inactivated viruses or CPG-ODN (8). Incubation of GM-DCs with active MVA induced IFN- $\alpha$ and IL- 6 production in WT and TLR9-deficient cells, demonstrating the TLR9-independent recognition of active MVA. No IFN- $\alpha$ production and no IL- 6 above constitutive levels produced was detected after incubation with UV-inactivated MVA (Figure 2C), potentially indicating that the TLR9-dependent recognition in response to MVA is not functional in those cells.

Recognition of ECTV but not of MVA exclusively depends on TLR9 and $p D C$. To define the individual activation profiles of the 2 main DC subsets among the FL-DCs, we sorted pDCs and cDCs, infected them with ECTV and MVA, and measured the IFN- $\alpha$ and IL- 6 production. WT pDCs produced IFN- $\alpha$ to both ECTV and MVA and very little IL-6 to ECTV. However, cDCs or TLR9-deficient pDCs produced IFN- $\alpha$ in response only to MVA but not ECTV (Figure 2D). WT and TLR9-deficient cDCs also produced large amounts of IL- 6 in response to MVA but not to ECTV. These results support the observations obtained with DC maturation (Figure 2A) and demonstrate that effective recognition of ECTV depends on the presence of TLR9, and in particular that IFN- $\alpha$ production by

\section{Figure 3}

Survival of WT and TLR9-deficient mice in response to ECTV infection. WT (A) and TLR9-deficient mice (B) were infected intranasally with varying doses of ECTV (TCID 50 per mouse) as indicated, and survival was monitored for at least 4 weeks. The experiments were performed with the indicated numbers of mice, and data represent at least 3 individual experiments for each viral dose in WT mice $(\mathbf{A})$ or 7 experiments for the dose of 100 for TLR9-KO mice (B) and 1 experiment for the other doses (B). The data for the dose of $1 \times 10^{4}$ in WT (A) and 100 in TLR9-KO mice (B) include control mice of other experiments. p.i., after infection.

ECTV is dependent upon pDCs. ECTV clearly lacks recognition via other TLR9-independent pathways. On the other hand, recognition of MVA by both pDCs and cDCs involves an additional TLR9independent mechanism.

To analyze ex vivo isolated pDC-containing cell populations in addition to in vitro generated FL-DCs, we stimulated WT and TLR9-deficient total bone marrow cells, a rich source of pDCs in vivo, with active or UV-inactivated MVA in parallel to CpG-ODN as a control. Similar to our results with FL-DCs, active MVA induced robust IFN- $\alpha$ production in WT and TLR9-deficient bone marrow cells, whereas with the lack of TLR9, the IFN- $\alpha$ production in response to UV-inactivated MVA and CPG-ODN was completely abrogated (Figure 2E). Thus, these data demonstrated that MVA was recognized by freshly isolated bone marrow cells via a UV-sensitive TLR9-independent pathway and a TLR9-dependent pathway.

TLR9-deficient mice have a drastically increased susceptibility to ECTV infection. Previous reports have clearly demonstrated the recognition of DNA viruses by TLR9 in vitro, but the in vivo relevance of TLR9 for the survival of mice is less clear. TLR9-deficient mice showed either no difference in survival in infection models using HSV; or limited survival differences within a narrow range in infection models using mouse CMV (MCMV) (9-11). Given the lack of TLR9-independent recognition in vitro by ECTV (Figure 2, A and D), we hypothesized that TLR9 would be an important factor for the survival of ECTV infection. Similar to VARV infection in humans, ECTV is highly species specific and is a natural mouse pathogen, able to effectively infect via the respiratory tract after exposure with only small viral doses. Thus, we applied ECTV via the intranasal route.

Initial experiments using relatively high doses of ECTV $\left(1 \times 10^{4}\right.$ $50 \%$ tissue culture infective doses $\left.\left[\mathrm{TCID}_{50}\right]\right)$ demonstrated that TLR9-deficient mice died at least 2 days earlier than WT mice. To further evaluate the susceptibility and quantify the $\mathrm{LD}_{50}$, we infected TLR9-deficient and WT mice with varying doses of ECTV. All

\section{Figure 4}

MVA protects WT mice if given simultaneously with lethal doses of ECTV. WT mice were infected intranasally with lethal doses of ECTV as indicated and simultaneously inoculated intranasally with (black circles) or without (gray squares) $1 \times 10^{8} \mathrm{TCID}_{50}$ of MVA, and survival was monitored for 4 weeks. The experiments were performed with the indicated numbers of mice, and data represent the results of 2 individual experiments.

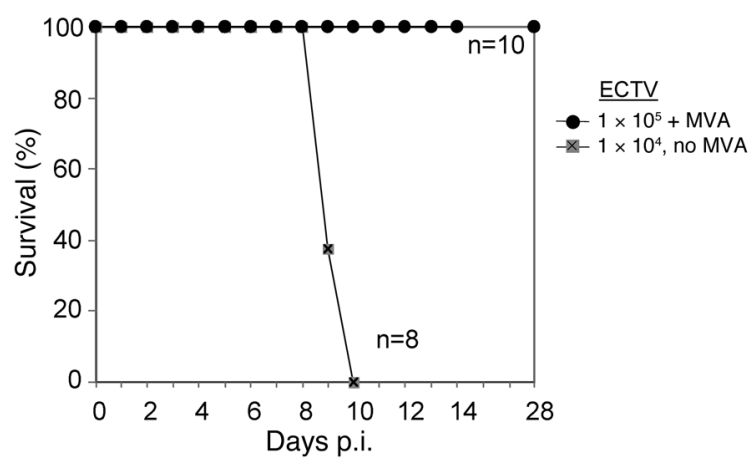




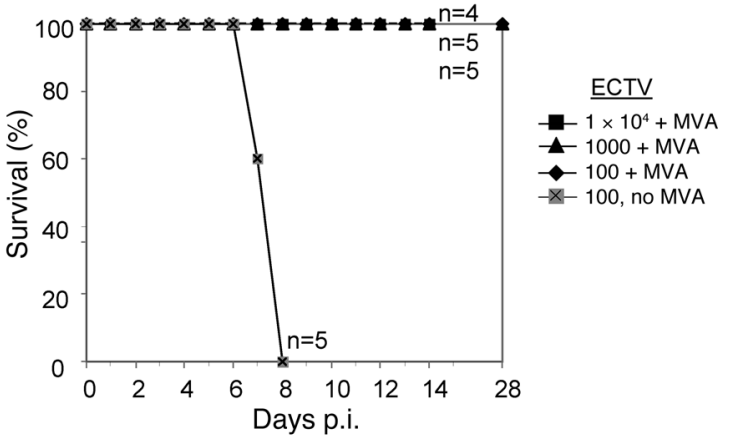

Figure 5

MVA protects TLR9-deficient mice if given simultaneously with lethal doses of ECTV. TLR9-deficient mice were infected intranasally with lethal doses of ECTV as indicated and simultaneously inoculated intranasally with (black symbols) or without (gray squares) $1 \times 10^{8} \mathrm{TCID}_{50}$ of MVA, and survival was monitored for 4 weeks. The experiment was performed with the indicated numbers of mice.

TLR9-deficient mice died after infection with as little as $30 \mathrm{TCID}_{50}$, whereas none died after inoculation with $10 \mathrm{TCID}_{50}$ (Figure 3B). In contrast, none of the WT mice died after infection with 100 TCID $_{50}$, and only when $1 \times 10^{4} \mathrm{TCID}_{50}$ was used did all mice succumb to ectromelia infection (Figure 3A). We found some variation between experiments with WT mice using the doses of 300-3,000 TCID ${ }_{50}$, which was partially sex specific, with male mice being more susceptible than female mice. We calculated an $\mathrm{LD}_{50}$ of $19 \mathrm{TCID}_{50}$ for the TLR9-deficient mice and an $\mathrm{LD}_{50}$ of about 2,120 $\mathrm{TCID}_{50}$ for the WT mice. Thus, TLR9-deficient mice were more than 100-fold more susceptible to ECTV infection than WT mice. We therefore conclude that in strong agreement with the in vitro data, TLR9 is an essential component of the immune response to ECTV infection.

MVA immediately protects WT and TLR9-deficient mice from lethal ECTV challenge. Our in vitro experiments demonstrated that although ECTV could activate DCs, activation of DCs was entirely TLR9 dependent (Figure 2). However, as indicated by an increase in DC activation upon UV inactivation of ECTV (Figure 1), it also effectively suppressed some recognition by DCs. MVA, on the other hand, showed no obvious suppressive activity (Figure 1). We therefore hypothesized that MVA given at the same time as the pathogen would activate the immune system and as a result might induce immune responses that potentially control the pathogenic poxvirus. Indeed, MVA given at the same time or immediately after challenge with a high lethal dose of ECTV of $1 \times 10^{5}$ TCID $_{50}$ com-

\section{Figure 6}

MVA protects TLR9-deficient and WT mice against lethal ECTV challenge if applied subcutaneously. (A) TLR9-deficient mice were infected intranasally with 100 TCID $_{50}$ of ECTV and simultaneously inoculated subcutaneously with (black squares) or without (gray squares) $1 \times 10^{8}$ $\mathrm{TCID}_{50}$ of MVA. (B) WT mice were infected intranasally with $1 \times 10^{4}$ $\mathrm{TCID}_{50}$ of ECTV and simultaneously inoculated subcutaneously with $1 \times 10^{8} \mathrm{TCID}_{50}$ of MVA (black squares) or with the corresponding amount of $1 \times 10^{8} \mathrm{TCID}_{50}$ of UV-inactivated CVA (black triangles). Survival was monitored for 4 weeks. The experiments were performed with the numbers of mice as indicated, and data represent the results of 2 individual experiments for WT mice with MVA and 1 experiment for WT mice with UV-inactivated CVA or for TLR9-deficient mice with MVA pletely protected WT mice against death, whereas all control mice died with the 10 -fold-lower dose of $1 \times 10^{4} \mathrm{TCID}_{50}$ (Figure 4).

Since MVA induced a strong TLR9-independent activation of immune cells in vitro, we next tested whether MVA could protect TLR9-deficient mice against ECTV infection. Similar to the protection seen in WT mice (Figure 4), MVA immediately protected TLR9-deficient mice against highly lethal doses of ECTV infection. Whereas all untreated control mice died with 100 TCID $_{50}$, all MVAtreated mice survived a challenge even with $1 \times 10^{4} \mathrm{TCID}_{50}$, a dose that exceeds by 500 -fold the $\mathrm{LD}_{50}$ for TLR9-deficient mice (Figure 5). We observed that TLR9-deficient mice challenged with high doses of $\operatorname{ECTV}\left(3,000\right.$ and $1 \times 10^{4}$ TCID $\left._{50}\right)$ developed tail lesions after 2-3 weeks that disappeared after 4 weeks (data not shown). The tail lesions on otherwise symptom-free TLR9-deficient mice indicated that MVA-induced immune responses were able to prevent severe ECTV-induced disease and death but not to completely eliminate the virus within the first weeks.

Mice can be protected against lethal ECTV infection if MVA is applied to a different site. To ascertain whether the immediate protection in WT and TLR9-deficient mice was absolutely dependent on the coadministration of MVA to the same site as the ECTV infection, we challenged mice intranasally with a lethal dose of ECTV and applied MVA via a subcutaneous injection. The TLR9-deficient (Figure 6A) and WT mice (Figure 6B) survived the lethal ECTV infection, without any signs of sickness, if MVA was applied to the subcutaneous site (Figure 6). Thus coadministration of MVA to the same site as ECTV was not essential for immediate protection.

Inactivated orthopoxviruses are less efficient than MVA in protection from lethal ECTV infection. Our in vitro experiments have suggested that UV-inactivated orthopoxviruses act as exclusive TLR9 agonists but have lost their ability to stimulate via a TLR9-independent way (Figure 2). To test whether this "TLR9-only" stimulation in the presence of orthopoxvirus antigen would mount any protection, we challenged WT mice with a lethal dose of ECTV and applied subcutaneously the equivalent of $1 \times 10^{8} \mathrm{TCID}_{50}$ of a UV-inacti-
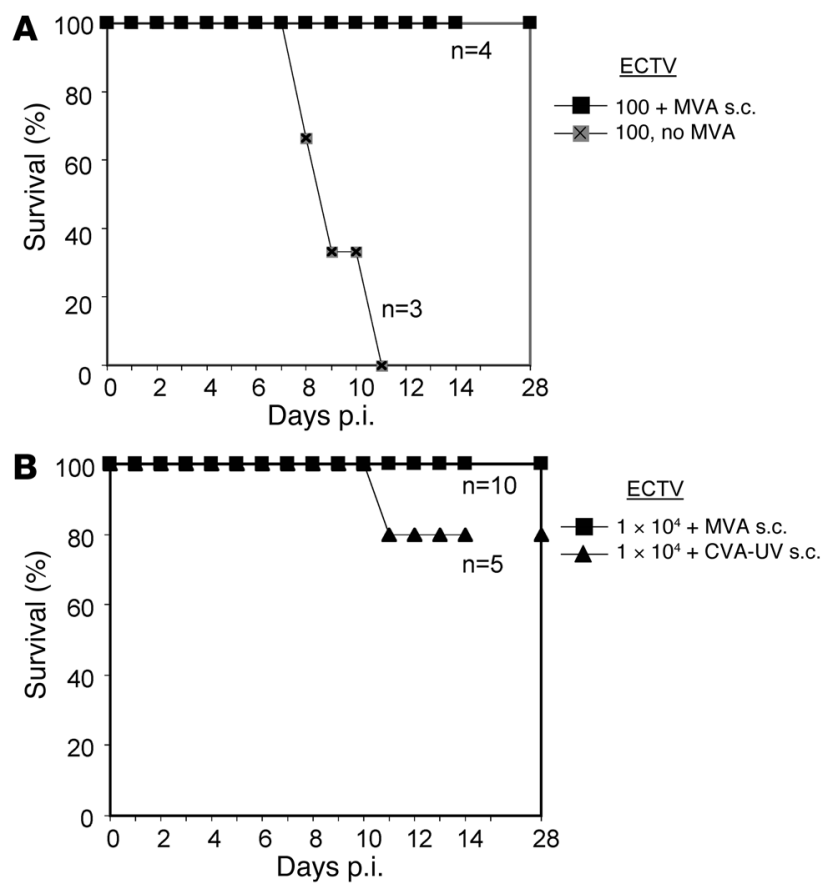


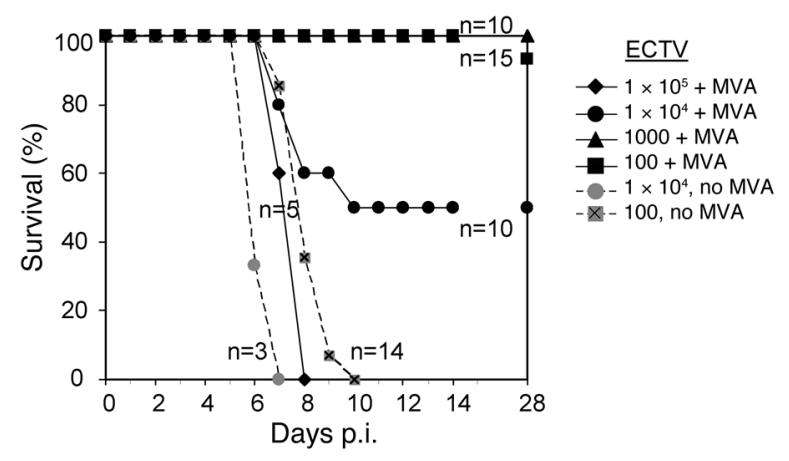

Figure 7

MVA partially protects IFN-I-R-deficient mice if given simultaneously with lethal doses of ECTV. IFN-I-R-deficient mice were infected intranasally with lethal doses of ECTV as indicated and simultaneously inoculated intranasally with (black symbols) or without (gray symbols) $1 \times 10^{8} \mathrm{TCID}_{50}$ of MVA, and survival was monitored for 4 weeks. The experiments were performed with the indicated numbers of mice, and data represent the results of at least 2 individual experiments for the challenge dose of 100 and $1,000 \mathrm{TCID}_{50}$ or 1 experiment for the challenge dose of $1 \times 10^{4}$ and $1 \times 10^{5} \mathrm{TCID}_{50}$.

vated CVA. Of the 5 mice challenged, 1 died on day 11, whereas the others survived (Figure 6B). However, in contrast to the mice that received the same dose of active MVA subcutaneously, all mice treated with inactivated CVA showed strong signs of sickness including lethargy, and they developed tail lesions that healed only in the fourth week of challenge. Thus, inactivated orthopoxviruses, although providing viral antigen and potential TLR9 ligand, seem to induce protection that is inferior to the robust protection achieved with active MVA.

MVA-mediated immediate protection from lethal ECTV challenge is partially independent of IFN-I. To elucidate whether administration of MVA was able to immediately protect other immunocompromised mice and to shed light on the mechanism of protection, we performed experiments with IFN-I receptor-deficient (IFN-I-R-deficient) mice, which cannot respond to type I IFN and are known to be highly susceptible to several viral infections, including poxvirus infections (18). Our initial experiments demonstrated that similar to TLR9-deficient mice, all IFN-I-R-deficient mice died after challenge with 100 TCID $_{50}$ of ECTV. Since the IFN- $\alpha$ production in vitro to ECTV but not to MVA was dependent on TLR9, we hypothesized that MVA-induced IFN- $\alpha$ was an essential part of the immediate protection in TLR9-deficient mice. However, whereas the untreated control IFN-I-R-deficient mice died with a challenge of 100 TCID $_{50}$ ECTV within 10 days, immediate MVA treatment surprisingly protected the IFN-I-R-deficient mice against a challenge with 100 and 1,000 TCID $_{50}$ ECTV (Figure 7). Of a total of 15 IFN-I-R-deficient mice challenged with 100 TCID $_{50}$ of ECTV, 1 mouse developed a swollen limb and had to be euthanized after 3 weeks for ethical reasons, whereas the other 14 mice and all 10 mice challenged with 1,000 ECTV were free of symptoms for more than 4 weeks. However, with higher doses of ECTV, the protection of IFN-I-R-deficient mice was much less robust. Half of the IFN-I-Rdeficient mice (5 of 10 mice) challenged with $1 \times 10^{4}$ ECTV died, and all IFN-I-R-deficient mice challenged with $1 \times 10^{5}$ ECTV died (Figure 7). Since these higher doses correspond to viral challenges that WT mice on the same background could survive in the presence of MVA, we conclude that one mechanism of the immediate protection via MVA is mediated by IFN-I. However, some protection is clearly mediated via IFN-I-independent mechanisms, since MVA could protect mice against low and intermediate doses of lethal ECTV infection even in the absence of a functional IFN-I system.

The immediate protection via MVA in ECTV infection depends on adaptive immune responses. MVA is known to induce strong adaptive immune responses including CTL responses and antibody formation, which both contribute to protection against pathogenic orthopoxviruses (19-21). Previously we and others have shown that TLR9-deficient mice are able to mount stable CTL and antibody responses upon DNA vaccination, thus demonstrating the overall ability of these mice to mount solid adaptive immune responses $(22,23)$.

To test whether the absence of TLR9 would affect adaptive immune responses to poxviruses, we applied MVA and measured antibodies against poxviruses by ELISA in the serum- and poxvirusspecific CTL responses by pentamer staining of B8-specific CTLs in spleen cells and peripheral blood cells. TLR9-deficient mice mounted robust poxvirus-specific antibody and CTL responses, indicating that adaptive immune responses in response to MVA vaccination are not dependent on the presence of TLR9 (data not shown).

We next investigated whether the measured adaptive immune responses would translate into long-lasting protection to ECTV infection, thus whether the MVA-induced protection in TLR9deficient mice was not only immediate (Figure 5) but also longlasting. Nine weeks after initial challenge, we rechallenged using $1 \times 10^{4} \mathrm{TCID}_{50}$ of ECTV the TLR9-deficient mice from the experiments described above (Figure 5) and in addition mice that had received MVA alone 9 weeks earlier. All TLR9-deficient mice that had received a single dose of MVA 9 weeks earlier either alone or in combination with ECTV survived the challenge with $1 \times 10^{4}$ TCID $_{50}$ of ECTV (data not shown). As observed with the immediate protection, the long-lasting protection of the TLR9-deficient mice after MVA treatment exceeded the $\mathrm{LD}_{50}$ by a factor of 500 . These experiments demonstrated that TLR9-deficient mice were capable of mounting and sustaining substantial protective immunity to poxvirus infection upon traditional vaccination with MVA, which most likely depended on adaptive immune responses.

To verify the role of adaptive immune responses in the immediate protection protocol, we challenged Rag $1^{-/-}$mice with ECTV

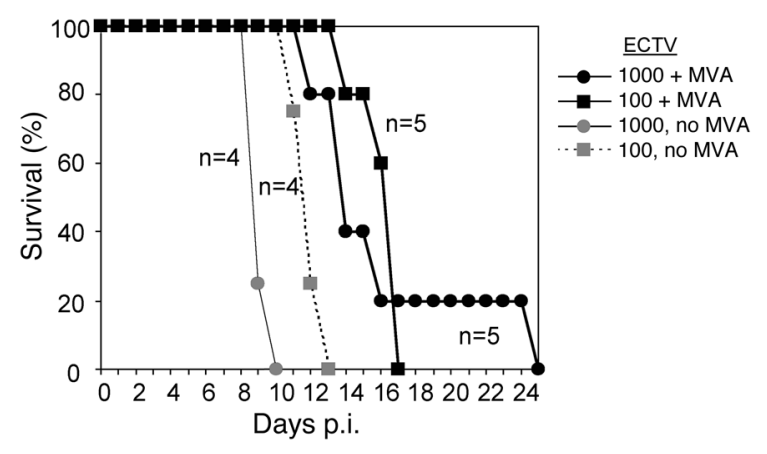

\section{Figure 8}

Long-term survival in response to ECTV infection even in the presence of MVA depends on adaptive immune responses. Rag $1^{-1-}$ mice were infected intranasally with doses of ECTV as indicated and simultaneously inoculated intranasally with $1 \times 10^{8} \mathrm{TCID}_{50}$ of MVA (black symbols) or without (gray symbols), and survival was monitored for 4 weeks. The experiment was performed with the indicated numbers of mice. 

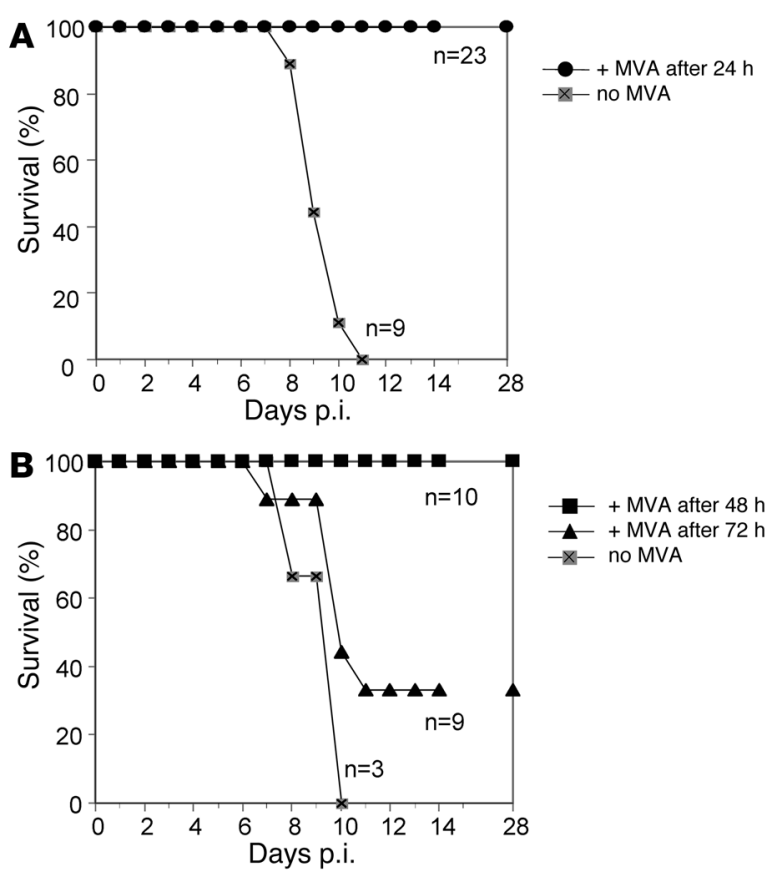

in the presence or absence of MVA (Figure 8). Rag1-/- mice lack mature $B$ cells and $T$ cells and thus are unable to produce antibodies and CTLs. Without coadministration of MVA, Rag $1^{-/-}$mice died rapidly in response to ECTV challenge (100 and 1,000 TCID 50 ). Cotreatment with MVA extended the survival of $\mathrm{Rag}^{-/-}$mice for several days, but all mice eventually died, demonstrating that adaptive immune responses are indeed crucial for the survival of ECTV even after immediate inoculation with MVA.

MVA fully rescues TLR9-deficient mice if applied 2 days after infection with ECTV. The WHO recommendation in cases of smallpox infection includes vaccination as quickly as possible after exposure. However, there exists only anecdotal historical information about the success of postexposure vaccination against smallpox, and in most cases, the prevaccination status of the individuals was not clear $(1,24)$. Moreover, in animal models, no significant survival benefit to postexposure vaccination was observed using as infection models either MPXV in monkeys or VACV in mice $(25,26)$.

Given this scenario and the fact that the intranasal infection model of ECTV is regarded as a good animal model for smallpox infection in humans, we analyzed whether the robust immediate protection against a lethal ECTV infection by MVA could be extended to a therapeutic postexposure intervention with MVA. As shown in Figure 9, MVA given up to 2 days after exposure to a lethal dose of ECTV completely protected TLR9-deficient mice against death, without any obvious signs of sickness (Figure 9 and data not shown). Some mice among the group that received MVA treatment as late as 3 days after a lethal dose of ECTV also survived (Figure 9B). To the best of our knowledge, these data are the first firm scientific data to show protection against death to speciesspecific orthopoxvirus infection using MVA or any other VACV as a postexposure treatment.

\section{Discussion}

The data presented here demonstrate that poxviruses variously activate DCs of mice. When total FL-DCs containing both pDCs

\section{Figure 9}

MVA therapeutically protects TLR9-deficient mice if applied after infection with a lethal dose of ECTV. TLR9-deficient mice were infected intranasally with $100 \mathrm{TCID}_{50}$ ECTV. After the indicated times of 24 hours (A) or 48 hours or 72 hours (B), the ECTV-infected mice were inoculated intranasally with $1 \times 10^{8} \mathrm{TCID}_{50}$ of MVA (black symbols) or without (gray squares), and survival was monitored for 4 weeks. The experiments were performed with the indicated numbers of mice, and data show the cumulative results of 3 individual experiments $(\mathbf{A})$ or 1 experiment $(\mathbf{B})$. Note that the 9 control mice in $\mathbf{A}$ include the 3 control mice in $\mathbf{B}$.

and cDCs are examined, it is clear that vaccinia virus CVA and CPXV are poor DC stimulators; ECTV is an intermediate stimulator; and MVA, SFV and CNPV are good DC stimulators. The stimulation of DCs in response to ECTV and particularly CVA and $\mathrm{CPXV}$ was greatly increased when these viruses were first UV inactivated, suggesting that these active viruses harbor mechanisms that inhibit DC recognition. Although MVA is a direct descendant of CVA (27), it was able to activate DCs and did not activate DCs more in a UV-inactivated state, suggesting that this virus has lost at least some of the inhibitory mechanisms of its CVA ancestor.

We have previously shown that other families of dsDNA viruses are detected via TLR9-dependent as well as TLR9-independent recognition pathways. To further examine the mechanisms of poxviral recognition by DCs, we studied the response to MVA and ECTV in more detail.

MVA, a highly attenuated VACV, was found to be recognized by DCs via both TLR9-dependent and TLR9-independent pathways (Figure 2). The pDCs clearly produced IFN- $\alpha$ in the presence and absence of TLR9. cDCs showed a strong IL- 6 response in both WT and TLR9-KO cells, with little difference in the presence or absence of TLR9. The response of DCs to UV-inactivated MVA was entirely dependent upon TLR9 expression (Figure 2B). This finding was similar to our previous findings with HSV-1, where active virus induced IFN- $\alpha$ in vitro in several DC subsets and macrophages independent of TLR9, whereas pDCs employed a TLR9-dependent pathway that also recognized inactivated HSV (8).

In sharp contrast to MVA, ECTV was not recognized at all by DCs that lacked TLR9. In fact recognition of ECTV via TLR9 was largely restricted to the pDCs (Figure 2). We hypothesize that the lack of TLR9-independent recognition of ECTV was due to an active inhibition. The increase in WT FL-DC activation upon UV inactivation of ECTV also suggests the possible existence of an additional mechanism that in part inhibits the TLR9-dependent recognition of ECTV.

The nature of TLR9-independent recognition in response to MVA is still elusive, as are the recognition pathways in response to other DNA viruses (28). However, recent reports rule out the absolute dependence on the presence of the TLR-associated adaptor molecules MyD88 and TRIF, as well as PKR (refs. 5, 14, and our unpublished observations). Similarly, the nature of the genes that inhibit the recognition of CVA, the ancestor of MVA, or those of ECTV, via TLR9-dependent or -independent pathways, are currently unknown. However, we have been able to demonstrate that the VACV products of $A 46 R$ and $A 52 R(4,29,30)$, previously implicated in the inhibition of TLR signaling molecules, are not responsible for this inhibition (data not shown).

The in vitro findings that showed absolute reliance on TLR9 for DC recognition of ECTV were clearly translated in vivo. TLR9deficient mice were more than 100-fold more susceptible to ECTV 
infection than WT mice (Figure 3). Other dsDNA virus infection models in TLR9-deficient mice have previously shown either no increase in susceptibility, as in the case of HSV-1 infections, or only minor susceptibility differences, as in the case of MCMV infections (9-11). Thus, this is the first example to our knowledge of the absolute requirement of TLR9 for survival against a viral infection.

Since MVA activated pathways and cells that ECTV did not activate and, as an orthopoxvirus, MVA also shared many ECTV antigens, we tested whether MVA could protect mice from lethal ECTV infection. Indeed, coinfection of MVA with ECTV prevented death of WT mice from ECTV infection. Moreover, the ability of MVA to activate cells via the TLR9-independent pathways was highlighted by the fact that this MVA-mediated immediate protection was also observed in mice lacking TLR9.

Mice that lack responsiveness to IFN-I were also highly susceptible to ECTV infection. The immediate protection protocol was applied to these mice, and with high-dose exposure, they were not protected to the same extent as WT mice, suggesting that IFN-I is part of the protection mediated by MVA in WT mice. However, IFN-I-R-deficient mice were protected against lower, but nevertheless lethal, doses of ECTV, clearly demonstrating that other mechanisms induced by MVA, are able to substitute for IFN-I during the innate phase of the immediate protection protocol. Of note, application of the traditional smallpox vaccine virus, Dryvax, instead of MVA, killed IFN-I-R-deficient mice even without ECTV challenge (data not shown). This finding was consistent with previous reports on lethality to this VACV in other immunocompromised mice (19).

Thus, both TLR9 and IFN-I are important facets in the innate protection of mice against ECTV infection. The factors other than IFN-I that are induced by MVA and important in the immediate protection protocol are the subject of ongoing studies. A role for other soluble factors (e.g., TNF- $\alpha$, IFN- $\gamma$ ) in protection against ECTV has previously been suggested $(2,3)$. Apart from soluble components, cellular innate mechanisms such as the induction of NK cells seem to play an important role during infections with poxviruses including ECTV infection (31). Determining which of these or possibly other mechanisms are involved in the MVA-mediated immediate protection will require further research.

The failure of the immediate protection protocol to induce sustained protection in the absence of adaptive immune responses (Figure 8) indicated that survival depended ultimately on adaptive immune responses. The prolongation of survival in the $\mathrm{Rag} \mathrm{1}^{-/-}$ mice also gave some indication of the duration of protecting innate mechanisms. Furthermore, it suggests that some early innate protecting mechanisms exist in the absence of adaptive immune cells, but as described previously for traditional vaccination strategies, survival to pathogenic poxvirus infection ultimately requires adaptive mechanisms to clear the virus. This prerequisite makes it unlikely that the induction of innate mechanisms - such as by application of IFN-I, TLR ligands, or other nonspecific innate stimuli - alone would suffice in the protection to lethal poxvirus infection if adaptive immune responses were not effectively triggered at the same time. Our experiment with UV-inactivated CVA (Figure 6B), which carries orthopoxvirus antigens and presumably activates only via TLR9, suggested that some limited protection could be achieved in immunocompetent mice. However, the fact that all mice at least became sick, in stark contrast to the mice treated with active MVA, which stayed symptom free, indicated that the protection via the active MVA is much more robust.
We then tested whether the MVA-mediated protection could be used in a therapeutic setting. Importantly, not only did MVA protect if given around the time of infection, but application as late as 2 days fully and 3 days partially protected mice from lethal ECTV infection. We could not find previous solid scientific evidence for postexposure vaccination in orthopoxvirus-naive individuals (24). Thus, this is the first demonstration to our knowledge of successful therapeutic vaccination against orthopoxvirus in naive individuals and, moreover, mice that are immunocompromised.

Given that therapeutic protection presumably requires a solid induction of innate immune mechanisms including antiviral cytokines to bridge the time for the adaptive immune responses to develop, therapeutic application of a noninhibitory orthopoxvirus such as MVA might also be beneficial in monkeys infected with MPXV. Indeed, Staib and colleagues have shown that mice were better protected with MVA than with VACV-Elstree if applied, at the latest, 2 days before challenge with VACV-WR (26). Thus, MVA seems to display protective advantages over VACV-Elstree in pathogenic orthopoxvirus infection models where the induction of innate immune mechanisms plays an important role. We tested whether VACV-Elstree would be inhibitory and found that DC maturation and cytokine production in vitro was inhibited, as seen with other VACV strains (data not shown). Thus, the in vitro findings of DC activation with different VACVs are likely translatable to other species infections.

This study defines TLR9 as an essential and in vivo highly relevant recognition molecule for poxviruses. Importantly, it provides evidence for the efficacy of using MVA as a conduit for immediate and therapeutic intervention against potential fatal poxvirus infection in healthy as well as immunocompromised individuals.

\section{Methods}

Mice. C57BL/6J mice were purchased from Harlan Winkelmann. TLR9deficient mice were generated on a $129 /$ Sv background and backcrossed to C57BL/ 6 mice for at least 8 generations as described previously $(6,8)$. Both the $129 / \mathrm{Sv}$ and C57BL/ 6 mouse strains are regarded as displaying a relatively high resistance to ECTV infection (32). However, to rule out that in our infection model the strain background would have an influence, we infected mice on the $129 / \mathrm{Sv}$ background with ECTV intranasally and found that indeed they displayed the relative resistant phenotype we saw in C57BL/6 mice, e.g., none of the mice died with the dose of $100 \mathrm{TCID}_{50}$, and the majority of mice even survived a dose of 3,000 (data not shown). IFN-I-R-deficient (A129) mice were originally obtained from Michel Aguet (University of Zurich, Zurich, Switzerland) (18) and backcrossed to C57BL/ 6 mice for 8 generations. Rag1 $1^{-/}$mice were purchased from the Jackson Laboratory and bred at the animal facility, Veterinary Faculty, University of Zurich.

Viruses. The MVA used for this study was MVA-BN, developed by Bavarian Nordic and deposited at European Collection of Cell Cultures (ECACC; V00083008). MVA was propagated and titered on primary chicken embryo fibroblasts (CEFs) that were prepared from 11-day-old embryonated pathogen-free hen eggs (Charles River Laboratories) and cultured in RPMI1640 medium supplemented with 10\% FCS. CVA and CNPV were kindly provided by A. Mayr (Veterinary Faculty, Ludwig-Maximilian University of Munich, Munich, Germany) and were propagated and titered on CEFs. ECTV strain Moscow and CPXV strain Brighton were obtained from ATCC as VR-1372 and VR-302, respectively, and were propagated and titered on Vero C1008 cells (ECACC 85020206). SFV was obtained from ATCC (VR-364) and propagated and titered on the rabbit cornea cell line SIRC obtained from ATCC (CCL-60). All cell lines were maintained in DMEM (Invitrogen) supplemented with 10\% FCS without antibiotics. All viruses 
used in animal experiments were purified twice through a sucrose cushion. For the UV inactivation of viruses, concentrated virus stocks were UV irradiated with a UV Chamber (Genelinker GS; Bio-Rad) for 15 minutes under sterilizing conditions. This treatment reduced the transduction efficiency of recombinant viruses below $2 \%$ of the original virus activity.

In vitro experiments. In vitro generated FL-DCs were generated and sorted essentially as described by us previously (8). In short, bone marrow cells were cultured in the presence of murine recombinant FL for 8 days. Resulting cells were greater than $90 \%$ CD 11 c positive, and $20 \%-60 \%$ of cells displayed the plasmacytoid phenotype (CD11 $\left.\mathrm{c}^{+} \mathrm{CD} 45 \mathrm{RA}{ }^{\text {hi }} \mathrm{B} 220^{\text {hi }} \mathrm{CD} 11 \mathrm{~b}^{\mathrm{lo}}\right)$. FL-DCs were either used unseparated or sorted into pDCs and cDCs using a FACS Aria instrument (BD Biosciences). In vitro generated GM-DCs were generated by culturing bone marrow cells in the presence of murine recombinant GM-CSF (Tebu-bio) as described previously (8). Cells were stained with antibodies specific for CD11c, B220, CD40, CD69, and CD86 (BD Biosciences). Propidium iodide $(1 \mu \mathrm{g} / \mathrm{ml})$ was included in the final wash to label dead cells. Flow cytometric analyses were performed on a FACSCalibur (BD Bioscience) and analyzed with WEASEL software (The Walter and Eliza Hall Institute for Medical Research, Melbourne, Victoria, Australia). Cell culture supernatants were harvested 18-24 hours after incubation with the viruses as indicated or with CpG-2216 $(0.5 \mu \mathrm{M}$ or $1 \mu \mathrm{M})$ as control in the presence of IL-3 and GM-CSF, and the secretion of IFN- $\alpha$ and IL- 6 was measured using commercially available ELISA reagents as described previously (8).

In vivo experiments and statistics. Mice were anesthetized with ketamine/ xylamine, and viruses were applied by intranasal dropwise installation in a total volume of $50 \mu \mathrm{l}$. ECTV dilutions as indicated were applied either alone or in combination with $1 \times 10^{8} \mathrm{TCID}_{50} \mathrm{MVA}$. Subcutaneous injections were performed in the inguinal region by applying a total of $1 \times 10^{8}$ $\mathrm{TCID}_{50}$ of MVA or a corresponding amount of UV-inactivated CVA by injecting 2 times a volume of $250 \mu \mathrm{l}$ each. The health status of infected mice was checked at least daily, and animals with grave symptoms of sickness or weight loss exceeding $25 \%$ were euthanized. All animal experiments were approved by the government of Upper Bavaria (Regierung von Oberbayern). For the calculation of the $\mathrm{LD}_{50}$, the Spearman-Karber method was used.

\section{Acknowledgments}

We thank Cornelia Wagner and other members of the research department and the members of the animal facility for their help and technical assistance. We thank the Process Development Department of Bavarian Nordic for producing purified viral stocks and Niels Wulff for his help with statistical analysis in calculating the $\mathrm{LD}_{50}$ of the mice.

Received for publication September 14, 2007, and accepted in revised form February 20, 2008.

Address correspondence to: Hubertus Hochrein, Research Immunology, Bavarian Nordic GmbH, Fraunhoferstr. 13, 82152 Martinsried, Germany. Phone: 49-89-8565-1334; Fax: 49-89-8565-1356; E-mail: hubertus.hochrein@bavarian-nordic.com.
1. Fenner, F., Henderson, D.A., Arita, I., Jezek, Z., and Ladnyi, I.D. 1988. Smallpox and its eradication. WHO. Geneva, Switzerland. whqlibdoc.who.int/ smallpox/9241561106.pdf.

2. Esteban, D.J., and Buller, R.M. 2005. Ectromelia virus: the causative agent of mousepox. J. Gen. Virol. 86:2645-2659.

3. Seet, B.T., et al. 2003. Poxviruses and immune evasion. Annu. Rev. Immunol. 21:377-423.

4. Bowie, A., et al. 2000. A46R and A52R from vaccinia virus are antagonists of host IL-1 and tolllike receptor signaling. Proc. Natl. Acad. Sci. U. S. A. 97:10162-10167.

5. Zhu, J., Martinez, J., Huang, X., and Yang, Y. 2007. Innate immunity against vaccinia virus is mediated by TLR 2 and requires TLR-independent production of IFN-beta. Blood. 109:619-625.

6. Hemmi, H., et al. 2000. A Toll-like receptor recognizes bacterial DNA. Nature. 408:740-745.

7. Wagner, H., and Bauer, S. 2006. All is not Toll: new pathways in DNA recognition. J. Exp. Med. 203:265-268.

8. Hochrein, H., et al. 2004. Herpes simplex virus type- 1 induces IFN-alpha production via Toll-like receptor 9-dependent and -independent pathways. Proc. Natl. Acad. Sci. U. S. A. 101:11416-11421.

9. Krug, A., et al. 2004. Herpes simplex virus type 1 activates murine natural interferon-producing cells through toll-like receptor 9. Blood. 103:1433-1437.

10. Delale, T., et al. 2005. MyD88-dependent and -independent murine cytomegalovirus sensing for IFNalpha release and initiation of immune responses in vivo. J. Immunol. 175:6723-6732.

11. Tabeta, K., et al. 2004. Toll-like receptors 9 and 3 as essential components of innate immune defense against mouse cytomegalovirus infection. Proc. Natl. Acad. Sci. U. S. A. 101:3516-3521.

12. Basner-Tschakarjan, E., et al. 2006. Adenovirus efficiently transduces plasmacytoid dendritic cells resulting in TLR9-dependent maturation and IFNalpha production. J. Gene Med. 8:1300-1306.

13. Lund, J., Sato, A., Akira, S., Medzhitov, R., and Iwasaki, A. 2003. Toll-like receptor 9-mediated recognition of Herpes simplex virus-2 by plasmacytoid dendritic cells. J. Exp. Med 198:513-520.

14. Waibler, Z., et al. 2007. Modified vaccinia virus Ankara induces Toll-like receptor-independent type I interferon responses. J. Virol. 81:12102-12110.

15. Engelmayer, J., et al. 1999. Vaccinia virus inhibits the maturation of human dendritic cells: a novel mechanism of immune evasion. J. Immunol. 163:6762-6768.

16. Drillien, R., Spehner, D., and Hanau, D. 2004 Modified vaccinia virus Ankara induces moderate activation of human dendritic cells. J. Gen. Virol. 85:2167-2175

17. Naik, S.H., et al. 2005. Cutting edge: generation of splenic CD8+ and CD8- dendritic cell equivalents in Fms-like tyrosine kinase 3 ligand bone marrow cultures. J. Immunol. 174:6592-6597.

18. Muller, U., et al. 1994. Functional role of type I and type II interferons in antiviral defense. Science. 264:1918-1921.

19. Wyatt, L.S., Earl, P.L., Eller, L.A., and Moss, B. 2004 Highly attenuated smallpox vaccine protects mice with and without immune deficiencies against pathogenic vaccinia virus challenge. Proc. Natl. Acad. Sci. U. S. A. 101:4590-4595.

20. Earl, P.L., et al. 2004. Immunogenicity of a highly attenuated MVA smallpox vaccine and protection against monkeypox. Nature. 428:182-185.

21. Stittelaar, K.J., et al. 2005. Modified vaccinia virus Ankara protects macaques against respiratory challenge with monkeypox virus. J. Virol. 79:7845-7851.

22. Spies, B., et al. 2003. Vaccination with plasmid DNA activates dendritic cells via Toll-like receptor 9
(TLR9) but functions in TLR9-deficient mice. J. Immunol. 171:5908-5912.

23. Babiuk, S., et al. 2004. TLR9-/- and TLR9+/+ mice display similar immune responses to a DNA vaccine. Immunology. 113:114-120.

24. Mortimer, P.P. 2003. Can postexposure vaccination against smallpox succeed? Clin. Infect. Dis. 36:622-629.

25. Stittelaar, K.J., et al. 2006. Antiviral treatment is more effective than smallpox vaccination upon lethal monkeypox virus infection. Nature. 439:745-748.

26. Staib, C., Suezer, Y., Kisling, S., Kalinke, U., and Sutter, G. 2006. Short-term, but not post-exposure, protection against lethal orthopoxvirus challenge after immunization with modified vaccinia virus Ankara. J. Gen. Virol. 87:2917-2921.

27. Meisinger-Henschel, C., et al. 2007. Genomic sequence of chorioallantois vaccinia virus Ankara, the ancestor of modified vaccinia virus Ankara. J. Gen. Virol. 88:3249-3259.

28. Ishii, K.J., and Akira, S. 2006. Innate immune recognition of, and regulation by, DNA. Trends Immunol 27:525-532.

29. Harte, M.T., et al. 2003. The poxvirus protein A52R targets Toll-like receptor signaling complexes to suppress host defense. J. Exp. Med. 197:343-351.

30. Stack, J., et al. 2005. Vaccinia virus protein A46R targets multiple Toll-like-interleukin-1 receptor adaptors and contributes to virulence. J. Exp. Med. 201:1007-1018.

31. Parker, A.K., Parker, S., Yokoyama, W.M., Corbett, J.A., and Buller, R.M. 2007. Induction of natural killer cell responses by ectromelia virus controls infection. J. Virol. 81:4070-4079.

32. Chaudhri, G., et al. 2004. Polarized type 1 cytokine response and cell-mediated immunity determine genetic resistance to mousepox. Proc. Natl. Acad. Sci. U. S. A. 101:9057-9062. 